\title{
ARTICLE \\ Oxytocin induces long-lasting adaptations within amygdala circuitry in autism: a treatment-mechanism study with randomized placebo-controlled design
}

\author{
Kaat Alaerts ${ }^{1}$, Sylvie Bernaerts ${ }^{1}{ }^{1}$, Jellina Prinsen (D) ${ }^{1}$, Claudia Dillen ${ }^{1}$, Jean Steyaert ${ }^{2}$ and Nicole Wenderoth ${ }^{3}$
}

Intranasal administration of the neuropeptide oxytocin (IN-OT) is increasingly explored as a potential treatment for targeting the core symptoms of autism spectrum disorder (ASD). To date, however, the impact of multiple-dose IN-OT treatment on human neural circuitry is largely unknown, and also the possibility that long-term IN-OT use may induce long-lasting neural adaptations remains unexplored. Using a double-blind, randomized, placebo-controlled, between-subject design (including 38 adult men with ASD), this treatment-mechanism study showed that 4 weeks of daily oxytocin administration (24 IU/day) significantly altered intrinsic (resting-state fMRI) functional connectivity of the amygdala to core regions of the "social brain" (particularly orbitofrontal cortex and superior temporal sulcus) up to 4 weeks and 1 year post treatment. The neural adaptations in functional coupling of the amygdala to the orbitofrontal cortex were associated with reduced feelings of avoidance toward others and-at the trend levelreduced repetitive behaviors. These observations contribute to a deeper mechanistic understanding of the neural substrates that underlie behavioral effects of multiple-dose IN-OT treatment, and provide initial insights into the long-lasting neural consequences of chronic IN-OT use on amygdala circuitry. Future studies are however warranted to further elucidate the long-term impact of INOT treatment on human neural circuitry and its behavioral consequences.

Neuropsychopharmacology (2020) 45:1141-1149; https://doi.org/10.1038/s41386-020-0653-8

\section{INTRODUCTION}

Autism spectrum disorder (ASD) is characterized by impairments in social communication and interaction, combined with restricted and repetitive behaviors and interests. To date, therapeutic interventions for ASD are mainly based on behavioral social skill trainings, since biomedical therapies or pharmacological interventions targeting social dysfunctions are largely unproven. However, in recent years, intranasal administration of the neuropeptide oxytocin (IN-OT) is increasingly explored as a potential treatment for elevating the core social problems characteristic of ASD (see recent review of IN-OT studies in ASD [1]).

OT is synthesized in magnocellular neurons of the supraoptic and paraventricular nuclei of the hypothalamus, and forms a key modulator of complex socio-affective responses, including affiliation, social approach and attachment, stress, and anxiety [2]. Mechanistically, OTs that have an impact on human brain function are suggested to involve a bottom-up anxiolytic effect to facilitate social approach behavior, and a top-down social salience effect to facilitate attention to, and perception of social signals [3-5]. These diverse psychosocial functions are at least in part mediated by the amygdala, which are known to receive direct axonal projections from hypothalamic nuclei, and show a high density of OT receptors for direct bottom-up neuroendocrine modulation of amygdala-centered circuits [6]. Furthermore, the distributed topography of the amygdala's connectional organization within the social brain places it in a central position to modulate a variety of brain networks that are important for social cognition [7].

From animal research, OT has been implicated in reducing amygdala reactivity through inhibitory GABAergic interneurons [8], a notion that is generally supported by human task-based functional magnetic resonance imaging (fMRI) studies showing mostly attenuated amygdala responses after a single dose of INOT in neurotypicals [9-20] and participants with ASD [21, 22] (but note several exceptions in neurotypicals $[23-26]$ and ASD $[27,28])$.

In addition to these task-based fMRI activation studies, also a handful of neuroimaging studies have explored the effects of INOT administration on patterns of amygdala connectivity to other brain regions. In one study, Kirsch et al. [13] identified reduced coupling of the amygdala to brainstem regions implicated in autonomic and behavioral manifestations of fear, therefore suggesting a mechanism by which IN-OT might attenuate emotional arousal. The amygdala is also anatomically and functionally connected to several areas of the frontal cortex (including the anterior cingulate gyrus (ACC), medial prefrontal cortex (MPFC), and orbitofrontal cortex (OFC)), which have been implicated in exerting a top-down modulatory influence over the amygdala to allow higher mental processes to regulate reactive emotionality [2]. To date, however, a heterogeneous pattern of results has emerged with respect to the effect of IN-OT on "intrinsic" functional connectivity of the amygdala to prefrontal

\footnotetext{
${ }^{1}$ Department of Rehabilitation Sciences, Group Biomedical Sciences, Neurorehabilitation Research Group, University of Leuven, KU Leuven, Belgium; ${ }^{2}$ Department of

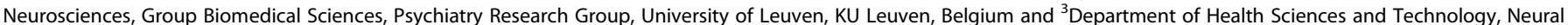
Control of Movement Lab, ETH Zurich, Zurich, Switzerland

Correspondence: Kaat Alaerts (Kaat.Alaerts@kuleuven.be)
}

Received: 3 December 2019 Revised: 31 January 2020 Accepted: 2 March 2020 Published online: 11 March 2020 
regions, as assessed with resting-state fMRI (see ref. [29] for a recent review). While some studies reported an increase in coupling between the amygdala and ACC and PFC regions, suggesting an enhancement of top-down neocortical regulation by IN-OT [11, 30-34], other studies identified reduced amygdala connectivity [11, 30, 32, 33, 35], or revealed no effect [31, 36, 37]. For example, differential effects of IN-OT have been reported for distinct subregions within the amygdala, with one study showing that increased amygdala connectivity to prefrontal regions was largely mediated by right SF and BLA subregions of the amygdala, whereas decreases in amygdala connectivity to temporal, occipital, and parietal regions were largely driven by CM subregions [32].

While these single-dose administration studies have provided important insights with respect to the acute effects of IN-OT $[29,38]$, the impact of multiple-dose IN-OT treatment on human brain function is largely unknown. In light of the growing number of trials assessing clinical responses of multiple-dose IN-OT treatment in ASD [39-44], it is crucial to gain a deeper mechanistic understanding into the neural substrates that underlie behavioral effects of multiple-dose IN-OT treatment. Indeed, the lack of mechanistic understanding and evaluation of potential longlasting neural consequences of chronic IN-OT use provides an important hurdle for the translation and further development of OT-based therapies.

Within the current study, a longitudinal, between-subject design was adopted to gain initial insights into the neural effects of multiple-dose IN-OT treatment (4 weeks of daily administrations) on "intrinsic" functional connectivity of amygdala-centered circuits as assessed with resting-state $\mathrm{FMRI}$, in adult men with ASD. To investigate whether repeated IN-OT administrations might induce long-lasting neural adaptations that outlast the period of actual administration, resting-state fMRI scanning was additionally performed at two follow-up sessions 4 weeks and 1 year post treatment.

\section{METHODS}

General study design

Within this study with double-blind, randomized placebocontrolled, between-subject design, we investigated the (longlasting) effects of multiple-dose IN-OT administration on intrinsic functional circuitry of the amygdala in young adult men with ASD. To do so, resting-state fMRI scanning was performed at baseline (T0), after 4 consecutive weeks of daily nasal spray administrations (T1), and at two follow-up sessions, 4 weeks (T2) and 1 year after cessation of the treatment (T3) (see Fig. 1, CONSORT flow diagram for the number of participants included in each assessment session). The neural assessments were conducted at the Leuven University Hospital in the context of a larger study (registered at the EU Clinical Trials register: Eudract 2014-000586-45 and clinicaltrial.gov: NCT02940574) $[45,46]$. Written informed consent was obtained from all participants prior to the study. Consent forms and study design were approved by the local Ethics Committee for Biomedical Research at the University of Leuven, KU Leuven (S56327) in accordance with The Code of Ethics of the World Medical Association (Declaration of Helsinki).

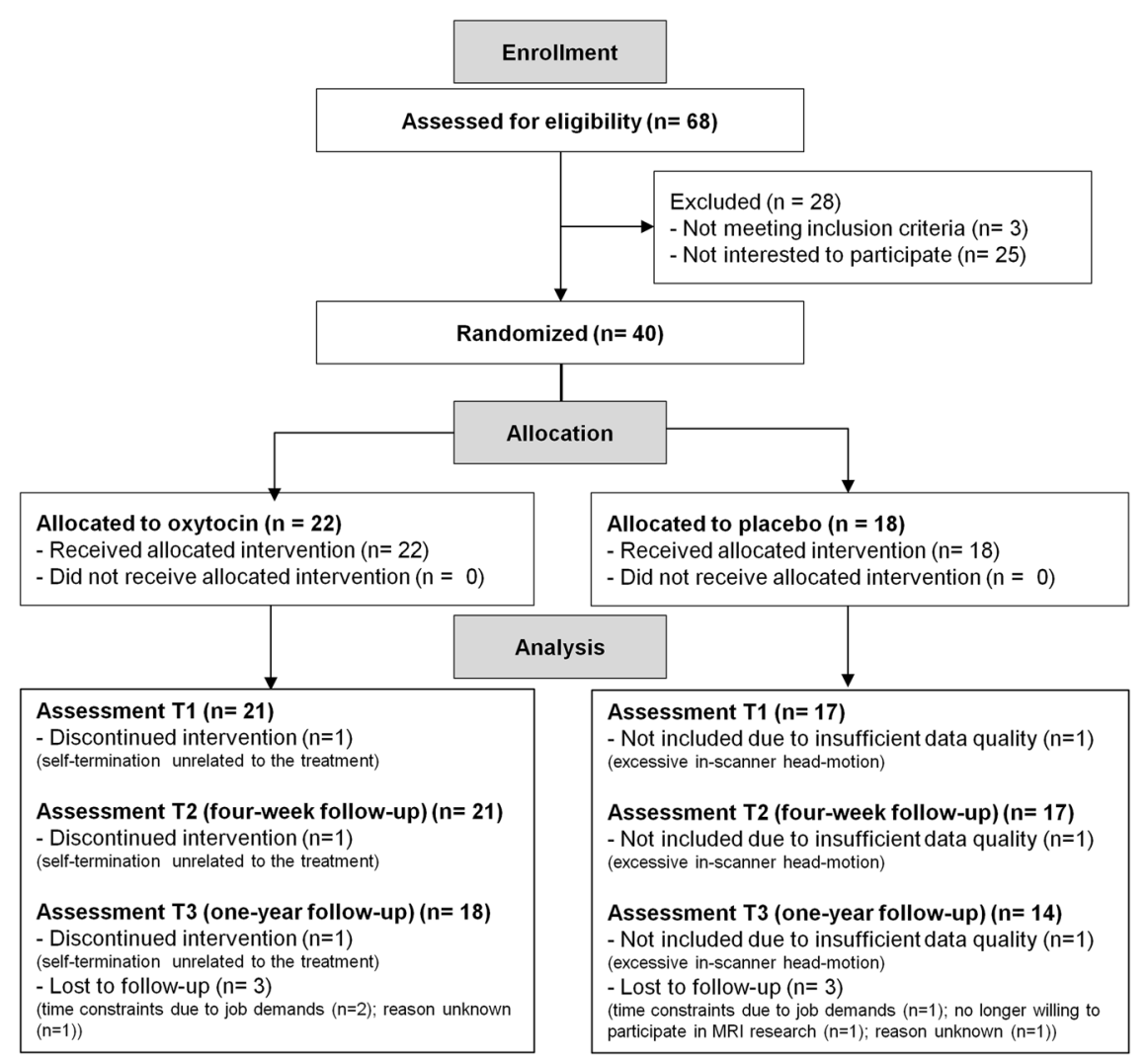

Fig. 1 CONSORT flow diagram. MRI scanning was performed at baseline (T0), after the 4-week (oxytocin/placebo) treatment (T1), and at two follow-up sessions, 4 weeks (T2) and 1 year after cessation of the treatment (T3). One participant of the oxytocin group discontinued the intervention after 1 week due to self-termination (unrelated to the treatment). One patient of the placebo group completed the 4-week treatment, but was excluded from the MRI analyses due to excessive in-scanner head motion (mean framewise displacement exceeding $0.5 \mathrm{~mm}$, requiring removal of $98.5 \%$ of images). Six additional participants (three oxytocin and three placebo) were lost for the follow-up session T3. 
Table 1. Demographic and clinical characteristics of participants randomized to receive oxytocin or placebo.

\begin{tabular}{|c|c|c|c|c|}
\hline & $\begin{array}{l}\text { Oxytocin } \\
N=21\end{array}$ & $\begin{array}{l}\text { Placebo } \\
N=17\end{array}$ & $t$ Value & $p$ \\
\hline Age & $24.76 \pm 4.85$ & $24.06 \pm 5.54$ & 0.42 & 0.68 \\
\hline \multicolumn{5}{|l|}{ IQ } \\
\hline Total IQ & $101.76 \pm 12.52$ & $107.29 \pm 18.91$ & -1.08 & 0.29 \\
\hline \multicolumn{5}{|l|}{$A D O S$} \\
\hline Total & $7.19 \pm 4.312$ & $7.59 \pm 3.89$ & -0.29 & 0.77 \\
\hline Communication & $2.14 \pm 1.35$ & $2.24 \pm 1.44$ & -0.20 & 0.84 \\
\hline Social interaction & $5.05 \pm 3.41$ & $5.35 \pm 3.14$ & -0.28 & 0.78 \\
\hline RRB & $1.19 \pm 1.29$ & $1.06 \pm 0.9$ & 0.36 & 0.72 \\
\hline
\end{tabular}

\section{Participants}

Participants were mainly recruited from the Autism Expertise Centre at the Leuven University Hospital between April 2015 and December 2016. Diagnosis of an ASD was made by a multidisciplinary team based on the strict criteria of the DSM-IV-TR (Diagnostic and Statistical Manual of Mental Disorders). Prior to the treatment, the ADOS (Autism Diagnostic Observation Schedule) [47] and estimates of intelligence (6-subtest short version of the Wechsler Adult Intelligence Scale-IV-Dutch version) were acquired from all participants. Baseline symptom severity (ADOS), IQ scores, and age were not significantly different between participants randomized to receive the OT or PL treatment (Table 1).

Inclusion/exclusion criteria. Inclusion criteria comprised clinical diagnosis of ASD, gender (male), and age (18-35 years old). Exclusion criteria for participation comprised any neurological disorder (e.g., stroke, epilepsy, and concussion), demonstrated genetic disorder, or any contraindication for MRI. Current psychoactive medication use and the presence of comorbid psychiatric disorders were screened (Table 1 and Supplementary Table 1). Handedness was assessed based on self-report.

Sample size. In one prior randomized placebo-controlled clinical trial, a crossover design was used to assess the effects of multipledose OT treatment ( 6 weeks of twice-daily doses) on resting-state fMRI functional connectivity in 20 adults with ASD, and significant effects (large-size) were reported for a total of 17 participants who completed the OT/PL crossover treatment [43]. Considering this prior crossover study and the lack of other prior studies assessing chronic neural effects using parallel designs, the current sample size was set at a comparable sample size.

Intervention

Participants were randomly assigned to receive the OT or PL treatment based on a computer-generated randomized order. Except for the manager of randomization, all research staff conducting the study, participants, parents, and partners were blind to treatment allocation. OT (Syntocinon ${ }^{\circledR}$, Sigma-tau) and placebo (saline natrium-chloride solution) were administered in amber $15-\mathrm{ml}$ glass bottles with metered pump (ACA Pharma). Each puff per nostril contained 4 international units (IU) of OT.
Participants self-administered a once-daily dose of 24 IU (3 puffs/ nostril) in the morning over 4 consecutive weeks ( 28 doses in total). More detailed information on administration instructions, medication adherence, and side-effect screening is provided in ref. [46] and Supplementary Methods and Results.

MRI data acquisition and functional connectivity analysis MRI data acquisition. Anatomical and resting-state fMRI images (7 min, eyes open) were acquired on a 3.0 Tesla Philips MR scanner (Best, The Netherlands) with an eight-channel phased-array head coil at baseline (T0), after 4 weeks of daily IN-OT (24 IU/day) treatment (at least $24 \mathrm{~h}$ after the final administration) (T1), and at two follow-up sessions, 4 weeks (T2) and 1 year post treatment (T3). Detailed information on the scanning parameters, MRI data preprocessing, and in-scanner head motion analyses is provided in Supplementary Fig. 2 and Supplementary Methods and Results.

Functional connectivity of amygdala. In accordance with the neuroendocrine model in ref. [48], region-of-interest (ROI) connectivity analysis was performed by assessing treatmentinduced changes in connectivity between bilateral amygdala (seeds) and a distributed network of ROIs in the hypothalamus, brainstem, MPFC, anterior cingulate cortex (ACC), OFC, and posterior superior (pSTG) and middle temporal gyri (pMTG) (encompassing the posterior superior temporal sulcus pSTS) (see Fig. 2). All ROls were defined from the FSL Harvard-Oxford subcortical and cortical atlas, except for the hypothalamus, which was defined with a sphere centered on Montreal Neurological Institute (MNI) coordinates [xyz: $0,-4,-8]$ using a $12-\mathrm{mm}$ radius [49]. As implemented in the CONN functional connectivity toolbox 16.b (Whitfield-Gabrieli and Nieto-Castanon, 2012), mean time series were extracted by averaging across all voxels in each ROI, and bivariate correlation coefficients were computed between the time course of the amygdala seeds (left and right) and the time courses of the other ROls. Correlation values were Fisher z-transformed.

In addition to the hypothesis-driven analysis based on the neuroendocrine model in ref. [48], exploratory whole-brain analyses were performed by assessing treatment-induced changes in functional connectivity between bilateral amygdala (seeds) and all other regions of the cortical $(n=91)$ and 
subcortical ( $n=13$ ) Harvard-Oxford atlas, as well as the cerebellar parcellation of the AAL Atlas $(n=26)$. For completeness, additional exploratory analyses were performed by exploring the effect of IN-OT on whole-brain functional connectivity of distinct amygdala subregion-based ROls covering the centromedial (CM), basolateral (BLA), and superficial (SF) amygdala (defined using probabilistic maps from the SPM anatomy Toolbox as previously adopted in ref. [32]).

\section{Statistical analysis}

IN-OT treatment effect. To explore treatment-induced changes in amygdala functional connectivity, changes from baseline (T0) in connectivity scores were calculated for each connection (separately for each assessment session: T1-T3) and subjected to mixedeffect analyses with "subject" as random factor, and "treatment" (OT and PL), "session" (T1-T3), "amygdala seed" (left and right), and "connection" $(n=10)$ (hypothalamus, brainstem, MPFC, ACC, bilateral OFC, bilateral pSTG, and bilateral pMTG) as fixed factors. All statistics were performed with Statistica 13 (StatSoft. Inc., Tulsa, USA). The significance level was set at $p<.05$. Post hoc analyses were Bonferroni-corrected for multiple comparisons.

Treatment-induced changes in whole-brain amygdala connectivity were assessed separately for each assessment session (T1-T3) by subjecting change from baseline connectivity scores to independent $t$ tests (two-sided) with the between-group factor "treatment" (OT and PL). Considering the exploratory nature of the whole-brain analysis, amygdala connections displaying significant treatment effects are reported at an uncorrected $p<0.05$ threshold (connection-level, CONN toolbox).

Association with behavioral improvements. As reported in more detail in Bernaerts et al. [46], we previously explored behavioral improvements as a result of the 4-week IN-OT treatment in the same patient sample in terms of social functioning (social responsiveness scale-adult version: SRS-A [50]), repetitive behaviors (repetitive behavior scale-revised: RBS-R [51]), and attachment avoidance (state adult attachment measure: SAAM [52]).

In short, behavioral improvements were evident immediately after treatment (T1), and until 4 weeks (T2) and 1 year (T3) post treatment in repetitive behaviors (RBS-R) and feelings of avoidant attachment (SAAM). While the OT group also reported improvements in social symptoms (SRS-A), these improvements were not treatment-specific (i.e., comparable improvements were evident in the placebo group) (see Supplementary Table 2 and Bernaerts et al. [46] for a detailed description of treatment-induced changes in these behavioral measures).

Here, Pearson's $r$ correlation analyses were performed to explore relationships between treatment-induced changes in amygdala connectivity (mean across sessions T1-T3) and behavioral changes as reported previously [46] in terms of social functioning (SRS-A), repetitive behaviors (RBS-R), and attachment avoidance (SAAM). Considering the exploratory nature of these brain-behavior analyses, the results are reported at an uncorrected $p<0.05$ threshold.

\section{RESULTS}

Effect of IN-OT treatment on amygdala connectivity

Mixed-effect analyses revealed the main effect of treatment $(F(1$, $\left.35.87)=6.35 ; p=0.016 ; \eta^{2}=0.15\right)$, indicating that the OT group displayed an overall attenuation in amygdala connectivity compared with the PL group. However, this main effect needs to be interpreted in light of a significant treatment-by-connection interaction $\left(F(2,2004)=3.89 ; p<0.001 ; \eta^{2}=0.017\right)$, for which post hoc analyses (exploring treatment effects separately for each connection) showed that the attenuating effect of IN-OT on amygdala connectivity was most pronounced for connectivity to OFC (left and right) and right pSTG (all, $p<0.05$, Bonferronicorrected) (see Fig. 2). A similar trend was evident for
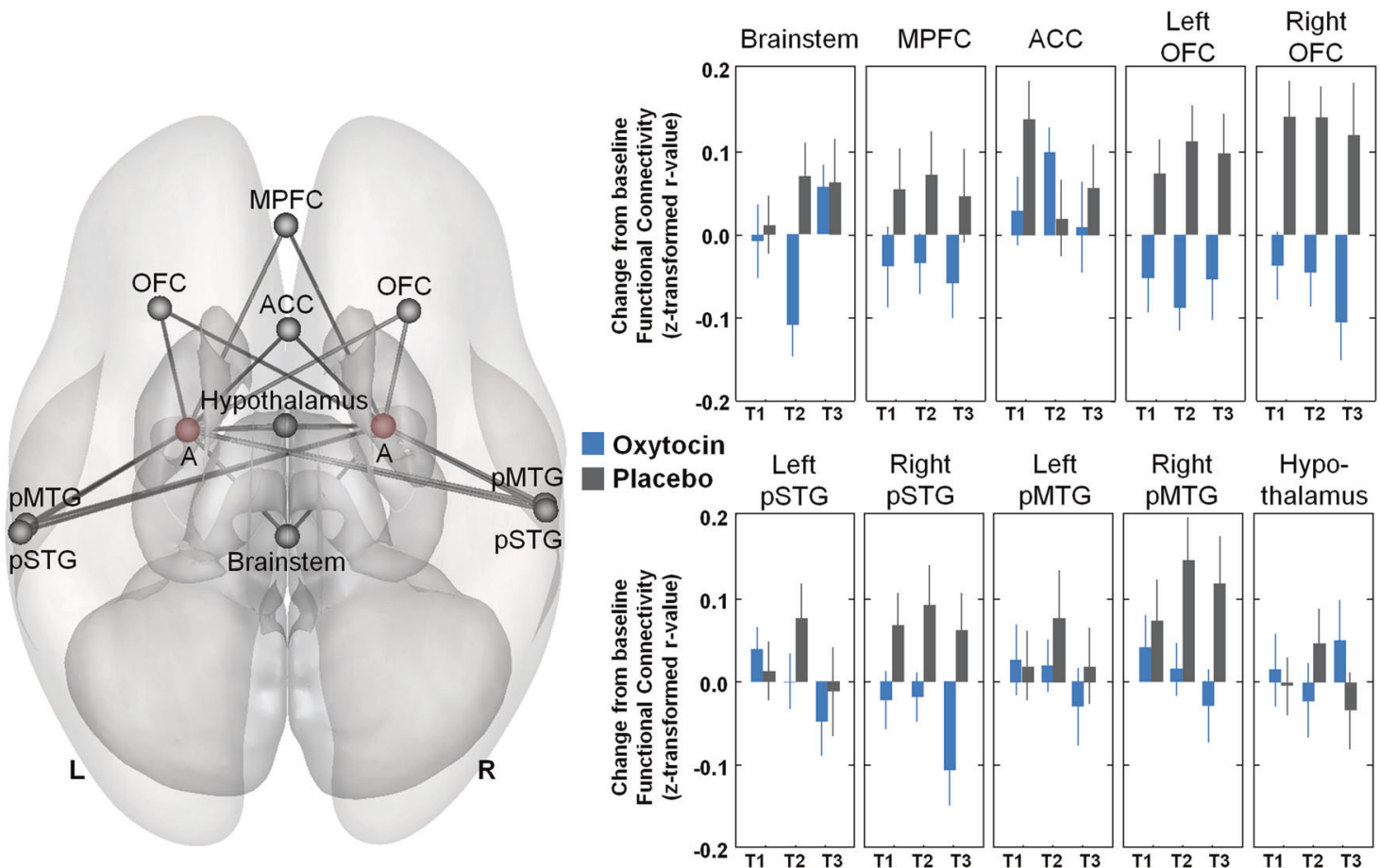

Fig. 2 Treatment-induced changes in amygdala connectivity. Treatment-induced changes in functional connectivity were explored between bilateral amygdala and a distributed network of regions of interest in the hypothalamus, brainstem, medial prefrontal cortex (MPFC), anterior cingulate cortex (ACC), orbitofrontal cortex (OFC), and posterior superior (pSTG) and middle temporal gyri (pMTG) (left panel). For each region, changes from baseline in amygdala connectivity are visualized separately for each treatment group (oxytocin and placebo) and assessment session (T1-T3). Vertical bars denote \pm standard errors. A amygdala, L left, R right. T1 assessment session immediately after the 4-week treatment (at least $24 \mathrm{~h}$ after the final administration), T2 assessment session 4 weeks post treatment, T3 assessment session 1 year post treatment. 
T1

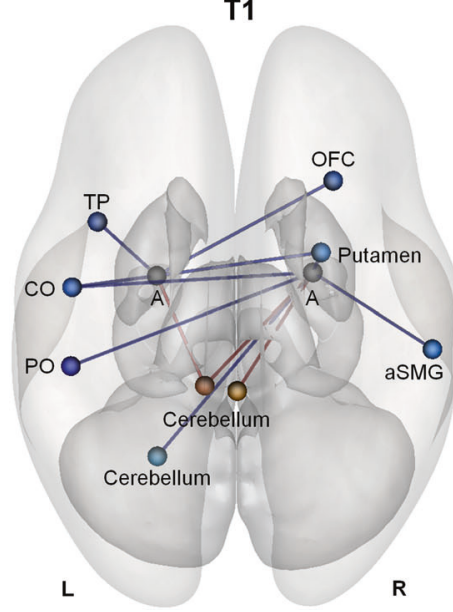

T2

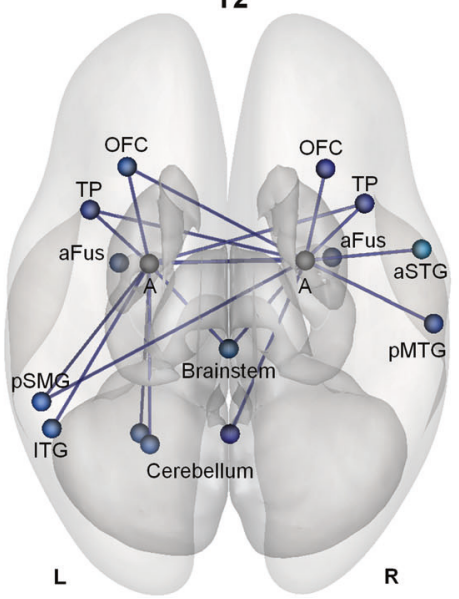

T3

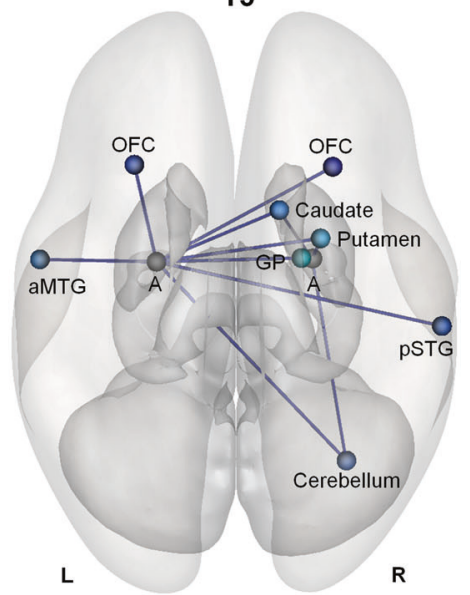

Fig. 3 Whole-brain amygdala connectivity analyses. Whole-brain analyses exploring treatment-induced changes in amygdala connectivity to other regions of the cortical $(n=91)$, and subcortical $(n=13)$ Harvard-Oxford Atlas, and the cerebellar parcellation of the AAL Atlas ( $n=26)$ revealed an overall pattern of reduced amygdala connectivity in the oxytocin group, compared with the placebo group. Connections displaying significant treatment effects (blue connections: oxytocin < placebo; red connections: oxytocin > placebo) are reported separately for each assessment session (T1-T3) at an uncorrected $p<0.05$ threshold (two-sided). T1 assessment session immediately after the 4-week treatment (at least $24 \mathrm{~h}$ after the final administration), T2 assessment session 4 weeks post treatment, T3 assessment session 1 year post treatment. A amygdala, PO parietal operculum cortex, CO central opercular cortex, aSMG anterior supramarginal gyrus, OFC orbitofrontal cortex, TP temporal pole, pMTG posterior middle temporal gyrus, aFus anterior fusiform cortex, pSMG posterior supramarginal gyrus, aSTG anterior superior temporal gyrus, ITG inferior temporal gyrus, pSTG posterior superior temporal gyrus, aMTG anterior middle temporal gyrus, GP globus pallidus, L left, $R$ right.

amygdala connectivity to the brainstem $\left(p_{\text {uncorrected }}<0.05\right)$, MPFC ( $\left.p_{\text {uncorrected }}<0.01\right)$, and right pMTG $\left(p_{\text {uncorrected }}<0.01\right)$, although these effects did not survive correction for multiple comparisons. No main effects of "amygdala seed", "session", or interactions with these factors were revealed (all $p>0.05$ ), indicating that the identified attenuations in amygdala connectivity were evident across the left and right amygdala regions and across the different assessment sessions (T1-T3). Figure 2 visualizes treatmentinduced changes in amygdala connectivity (averaged across the left and right amygdala seeds) for all connections.

In addition, exploratory whole-brain analyses were conducted by exploring treatment-induced changes in functional connectivity between bilateral amygdala (seeds) and all other regions of the cortical $(n=91)$ and subcortical $(n=13)$ Harvard-Oxford Atlas, as well as the cerebellar parcellation of the AAL Atlas $(n=26)$. The whole-brain analyses consistently identified a pattern of reduced amygdala connectivity immediately after the multiple-dose IN-OT treatment (T1), and at the 4-week (T2), and 1-year (T3) follow-up sessions (Fig. 3 and Supplementary Table 3 ). In addition to reductions in amygdala-OFC connectivity (identified at all sessions), reductions in amygdala connectivity were mainly identified with cortical regions in the temporal lobes (e.g., temporal pole (at sessions T1 and T2), middle and superior temporal gyrus (at sessions T2 and T3), and fusiform gyrus (session $\mathrm{T} 2)$ ), supramarginal gyrus (at sessions $\mathrm{T} 1$ and $\mathrm{T} 2$ ), as well as with regions in the cerebellum (at all sessions). Reductions in amygdala-subcortical connectivity were mainly identified for the brainstem (at session T2), putamen (at sessions T1 and T3), globus pallidus, and caudate (at session T3). Increases in amygdala connectivity were only identified immediately after the multipledose treatment (session T1) in terms of amygdala-cerebellum connectivity (Fig. 3 and Supplementary Table 3).

Further, as visualized in Supplementary Fig. 2, exploration of the effect of IN-OT on amygdala subregional functional connectivity also generally confirmed a pattern of attenuated amygdala connectivity, and additionally identified a pattern of reduced amygdala connectivity (CM subregion) to the hippocampal (T2) and parahippocampal structures (T1-T3), as well as to the insular cortex (BLA subregion: T1) (SF subregions: T1 and T2).

\section{Association between neural changes and behavioral improvements}

IN-OT-induced reductions in amygdala-OFC connectivity (mean across sessions $\mathrm{T} 1-\mathrm{T} 3$ ) were shown to be associated with selfreported improvements in feelings of avoidant attachment as assessed with the SAAM (mean improvement across T1-T3) $(r=$ $0.43 ; p=0.05$ ) (Fig. 4). Note however that no significant association was evident between treatment-induced changes in amygdala-OFC connectivity and baseline reports of avoidant attachment $(r=-0.25 ; p=0.28)$, indicating that neural treatment responses were not moderated by inter-individual differences in baseline attachment avoidance. At trend level, reductions in amygdala-OFC connectivity were also associated with improvements in repetitive behaviors (RBS-R) $(r=0.40 ; p=0.07)$ (Fig. 4), but not with improvements in social functioning (SRS-A) $(r=0.15$; $p=0.52$ ). No significant associations were evident between neural and behavioral changes in the PL group (all, $p>0.33$ ).

\section{DISCUSSION}

In this treatment-mechanism study, we identified neural changes in intrinsic functional connectivity of the amygdala after a multiple-dose treatment with IN-OT that outlasted the period of actual administration until 4 weeks and even 1 year post treatment. These findings provide initial indications that repeated administrations of IN-OT can induce long-lasting adaptations in amygdala-centered circuits in adult men with ASD.

The results converged on the identification of a relative decrease in intrinsic functional connectivity of the amygdala, with several regions of the socio-emotional processing circuit, and in particular with regulatory hubs in the OFC after multiple-dose IN-OT treatment. Whole-brain exploratory analyses and amygdala subregional analyses generally confirmed this pattern of results, showing overall reductions in amygdala connectivity to a 

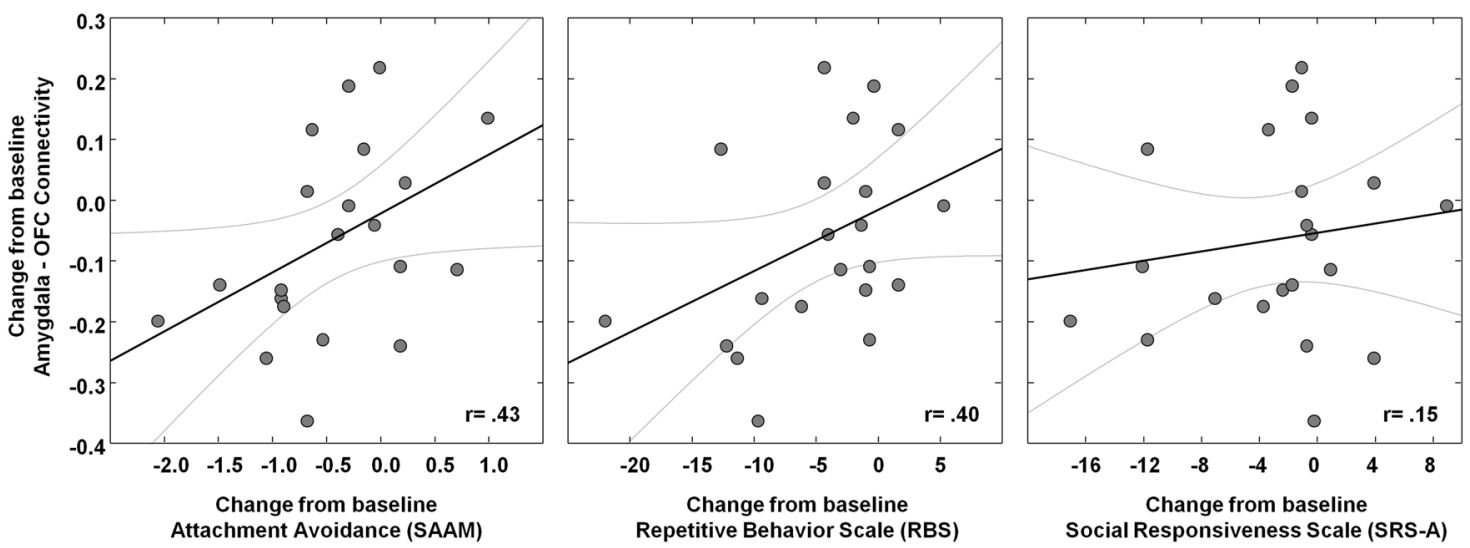

Fig. 4 Association between neural changes and behavioral improvements. Brain-behavior relationships are visualized between changes from baseline in amygdala connectivity (mean change across sessions T1-T3) and behavioral improvements (mean improvement across sessions T1-T3). Negative scores indicate pre-to-post behavioral improvement. OFC orbitofrontal cortex, SAAM state adult attachment measure. Gray lines denote $95 \%$ confidence bands.

distributed network of cortical (temporal cortex and supramarginal gyrus), subcortical (brainstem, putamen, and caudate), and cerebellar regions after multiple-dose treatment with IN-OT. These results indicate that after multiple-dose IN-OT treatment, slow spontaneous resting-state neuronal fluctuations in these regions became increasingly decoupled from the spontaneous neuronal fluctuations measured in amygdalar regions. Indeed, the IN-OT treatment primarily induced a reduction in the strength of coupling, rather than affecting the directionality of coupling (i.e., to anti-correlations) (see, e.g., Supplementary Fig. 3, visualizing the raw z-transformed $r$ values of the connectivity between amygdala and OFC).

From previous single-dose administration studies assessing the effect of IN-OT on resting-state functional connectivity, a mixed pattern of results has emerged, with some studies reporting an increase in coupling between the amygdala and top-down neocortical regulatory hubs in ACC and PFC regions after IN-OT [11, 30-34], while other studies identified reduced amygdala connectivity [11,30,32,33,35] or revealed no effect $[31,36,37]$ (see ref. [29] for a recent review). In line with the suggested top-down modulatory influence of prefrontal regions over the amygdala to allow higher mental processes to regulate reactive emotionality, identifications of increased amygdala-prefrontal coupling after IN-OT have been suggested to reflect enhanced top-down inhibitory control over amygdala reactivity, thereby facilitating attenuation of amygdala activation in response to aversive/threatening social stimuli (e.g., Labuschagne et al. [14]). The current observations of reduced connectivity between amygdala and prefrontal regions (OFC and at trend-level MPFC) after multiple-dose IN-OT are however at odds with this interpretation, and suggest that acute effects of IN-OT may be qualitatively different from chronically induced adaptations. While single-dose administrations may involve acute attenuations of amygdala reactivity (presumably due to enhanced top-down inhibitory control), it can be hypothesized that a recursive halting of excessive amygdala reactivity from repeated administrations of IN-OT may induce adaptations in amygdala-centered circuits, reflecting a reduced need for top-down regulatory modulation to effectively cope with excessive emotional reactivity. This interpretation is corroborated by the identified relationships between treatment-induced changes in amygdala-OFC coupling and behavioral improvements, indicating that reduced feelings of avoidance toward others (and at trend level, reductions in repetitive behaviors) were associated with stronger reductions in amygdala-OFC coupling.
Main and whole-brain exploratory analyses also identified treatment-related changes in amygdala coupling with (right) posterior STS-a key node of the "social brain" involved in social perception and theory-of-mind [53] —as well as with the brainstem. The observation of OT-induced reductions in amygdala-brainstem connectivity is in line with a previous neuroimaging study reporting a reduction in amygdala activation and amygdalabrainstem connectivity after a single dose of OT (during the presentation of fear-inducing visual stimuli) [13]. Descending projections from the amygdala to the brainstem are suggested to contribute to the regulation of the expression of autonomic reactions (arousal) to (socio-emotional) signals by modulating sympathetic-parasympathetic tone, and fear behavior [54]. The identification of a long-term impact (particularly at session T2) of multiple-dose OT treatment on "intrinsic" functional connectivity of the amygdala to the brainstem further underlines the importance of this circuitry in mediating the neural effects of OT. Recent metaanalyses of single-dose administration studies also consistently highlighted the middle and superior temporal gyri as robust areas of activation for mediating the (social) salience-enhancing effects of OT $[38,55,56]$. With the current study, we extend these findings by showing that the posterior STS also forms an important neural substrate for mediating the long-term, chronic effects of IN-OT treatment. This seems of particular relevance to ASD, since dysfunction of posterior STS regions (particularly right STS) has been highlighted as an important neural substrate for the socioemotional difficulties characteristic of ASD [57, 58].

Whole-brain exploratory analyses additionally identified changes in amygdala coupling to several subcortical nuclei, including putamen, caudate, and globus pallidus. These changes are anticipated to reflect IN-OT's interaction with basal ganglia regions for modulating reward-based learning and promoting approach-related motivational tendencies, especially during the processing of rewards related to interactions with others [59] (see recent review outlining the effects of single-dose IN-OT on basal ganglia structures [38]).

Finally, exploratory analyses also identified altered coupling between the amygdala and distinct regions in the cerebellum after multiple-dose IN-OT treatment. While the cerebellum has traditionally been associated with motor function, recent work points to a role for the cerebellum in higher affective and cognitive functions, and cerebellar pathophysiology has been documented in ASD [60]. In one prior single-dose IN-OT study, Eckstein et al. [32] reported increases in resting-state functional connectivity of bilateral amygdala with the contralateral cerebellum after IN-OT (i.e., left amygdala with right cerebellum). In the 
current study, both increased and decreased amygdala-cerebellar connectivity was identified immediately after the multiple-dose treatment (session T1). However, at the follow-up sessions, a predominant pattern of reduced amygdala-cerebellum connectivity was evident. Considering that the cerebellum is not typically considered a key region of the central oxytocinergic system, future studies are needed to elucidate its contribution in mediating the acute and chronic effects of IN-OT.

To date, studies investigating the chronic effects of multipledose IN-OT on neural circuitry are sparse [29]. In one study, Kovacs and Keri [61] examined resting-state amygdala connectivity in 41 individuals who reported "off-label" use of IN-OT purchased over the Internet (using 35 IU per dose, about four-and-a-half times a week over 9 weeks), and reported increased amygdala connectivity to dorsal ACC in the IN-OT group versus healthy controls, with a dose-dependent relationship between cumulative OT intake and connectivity strength [61]. These findings seem at odds with the current study, in which no significant treatment effects were identified in terms of amygdala-ACC connectivity (although we note a trend toward increased connectivity at session T2). However, the differential results might be driven by several apparent differences between studies in terms of study design (off-label use vs. randomized, placebo-controlled design), patient population, dose, and duration. In another multiple-dose study, Watanabe et al. [43] explored the effects of 6 weeks of IN-OT treatment on resting-state functional connectivity in adults with ASD, and reported an increase in connectivity between ACC and the prefrontal cortex [43]. In this study, it was noted that the effect size of the 6-week treatment was qualitatively similar to the effect size of a previous single-dose administration study [22], indicating that the multiple-dose treatment did not enhance functional connectivity above and beyond a single dose of IN-OT. One explanation for this similarity between the acute and chronic effect may be that participants in the 6-week study were scanned only 15 or 40 min after the last nasal spray administration, which corresponds to the optimal time frame for assessing acute, singledose effects of OT administration. As such, the possibility cannot be ruled out that the reported multiple-dose effect-at least in part-reflected an acute effect of exogenously administered OT. In contrast, in the current study, MRI scanning for assessing the multiple-dose effect (T1) was performed at least $24 \mathrm{~h}$ after the last OT administration, rendering the observed neural effects less susceptible to reflect an acute effect of IN-OT administration. Also, the neural changes at the 4-week (T2) and 1-year follow-up sessions (T3) are interpreted to solely reflect long-lasting adaptations in neural functioning due to IN-OT's recursive action on these circuits.

While the current study provides important new insights into the chronic effects of IN-OT on amygdala circuitry, several limitations need to be considered. First, although our sample size was comparable to that of prior multiple-dose administration studies, future studies with larger samples are warranted to corroborate the herein-identified long-term neural effects of IN-OT treatment. Second, in the current study, participants administered the OT nasal spray once a day (in the morning) (similar to ref. [39]), while the majority of prior multiple-dose OT studies administered two doses/day (one in the morning and one in the afternoon) (e.g., Watanabe et al. [43]). While elevated levels of OT have been demonstrated up to $7 \mathrm{~h}$ after a single-dose administration [62], future studies are needed to identify at what point in time the effects of intranasal administration of OT fade out, and when OT levels return back to baseline. Also, potential interactions with diurnal patterns of endogenous OT levels need to be explored to identify the most optimal dosing and timing of intranasal OT administrations. Furthermore, in light of the current observations of long-lasting neural effects, our study highlights that in addition to dose, future trials should be directed at identifying the optimal administration length and intervals, preferably adopting parallel, between-subject designs, as opposed to crossover designs with wash-out period. Also, the possibility that IN-OT's action may be further enlarged by simultaneously administering targeted behavioral therapies holds promise and should be addressed in future studies. Finally, similar to the present study, the majority of prior IN-OT studies in humans have been conducted with young adult men, which makes their generalizability across age and sex uncertain. This limitation is important to take into consideration since initial neuroimaging studies have provided indications that IN-OT may modulate neural and behavioral responses in a sexspecific manner. In a study by Ebner et al. [31], it was shown, for example, that the effect of IN-OT on amygdala-prefrontal connectivity varied depending on age and sex, indicating that, only in young women, IN-OT increased resting-state connectivity, but not in the other age/sex subgroups [31].

To conclude, our results showed that a 4-week multiple-dose INOT treatment induced long-term alterations in intrinsic functional connectivity of the amygdala, especially to regulatory regions in the OFC, which were associated with behavioral improvements in feelings of attachment and (at trend-level) repetitive behavior in young adult men with ASD. Since IN-OT is increasingly considered as a potential treatment for ASD, it is crucial to gain a deeper mechanistic understanding of the neural substrates that underlie behavioral effects of multiple-dose IN-OT treatment. While the current observations of long-term effects of IN-OT treatment on amygdala circuitry and associated behavioral improvements are promising and suggestive of a therapeutic potential of IN-OT for ASD, future studies are warranted to further elucidate the longterm impact of IN-OT treatment.

\section{FUNDING AND DISCLOSURE}

This research was supported by the Branco Weiss fellowship of the Society in Science-ETH Zurich and by grants from the Flanders Fund for Scientific Research (FWO projects KAN 1506716N, KAN $1521313 \mathrm{~N}$, and G079017N). SB and JP are supported by a fund of the Marguerite-Marie Delacroix foundation. The authors declare no competing interests.

\section{ACKNOWLEDGEMENTS}

We would like to thank Emmely Berra for her help in data collections. We would also like to thank all the participants of the study and our colleagues of the Leuven Autism Research Consortium (LAuRes).

\section{AUTHOR CONTRIBUTIONS}

$K A$, JS, and NW designed the study. SB and CD acquired the data. KA analyzed the data and wrote the paper. SB, JP, JS, and NW provided intellectual contribution to the data analyses and revised the paper. All authors read and approved the final paper.

\section{ADDITIONAL INFORMATION}

Supplementary Information accompanies this paper at (https://doi.org/10.1038/ s41386-020-0653-8).

Publisher's note Springer Nature remains neutral with regard to jurisdictional claims in published maps and institutional affiliations.

\section{REFERENCES}

1. Ooi YP, Weng SJ, Kossowsky J, Gerger H, Sung M. Oxytocin and autism spectrum disorders: a systematic review and meta-analysis of randomized controlled trials. Pharmacopsychiatry. 2017;50:5-13.

2. Meyer-Lindenberg A, Domes $G$, Kirsch $P$, Heinrichs $M$. Oxytocin and vasopressin in the human brain: social neuropeptides for translational medicine. Nat Rev Neurosci. 2011;12:524-38.

3. Kemp AH, Guastella AJ. Oxytocin: prosocial behavior, social salience, or approachrelated behavior? Biol Psychiatry. 2010;67:e33-4. 
4. Bartz JA, Zaki J, Bolger N, Ochsner KN. Social effects of oxytocin in humans: context and person matter. Trends Cogn Sci. 2011;15:301-9.

5. Shamay-Tsoory SG, Abu-Akel A. The social salience hypothesis of oxytocin. Biol Psychiatry. 2016;79:194-202.

6. Gimpl G, Fahrenholz F. The oxytocin receptor system: structure, function, and regulation. Physiol Rev. 2001;81:629-83.

7. Bickart KC, Dickerson BC, Barrett LF. The amygdala as a hub in brain networks that support social life. Neuropsychologia. 2014;63:235-48.

8. Huber D, Veinante P, Stoop R. Vasopressin and oxytocin excite distinct neuronal populations in the central amygdala. Science. 2005;308:245-8.

9. Domes G, Heinrichs M, Glascher J, Buchel C, Braus DF, Herpertz SC. Oxytocin attenuates amygdala responses to emotional faces regardless of valence. Biol Psychiatry. 2007;62:1187-90.

10. Eckstein M, Becker B, Scheele D, Scholz C, Preckel K, Schlaepfer TE, et al. Oxytocin facilitates the extinction of conditioned fear in humans. Biol Psychiatry. 2015;78:194-202.

11. Frijling $\mathrm{J}$, van ZM, Koch SB, Nawijn L, Veltman DJ, Olff M. Effects of intranasal oxytocin on amygdala reactivity to emotional faces in recently trauma-exposed individuals. Soc Cogn Affect Neurosci. 2016;11:327-36.

12. Gamer M, Zurowski B, Buchel C. Different amygdala subregions mediate valencerelated and attentional effects of oxytocin in humans. Proc Natl Acad Sci USA. 2010;107:9400-5.

13. Kirsch P, Esslinger C, Chen Q, Mier D, Lis S, Siddhanti S, et al. Oxytocin modulates neural circuitry for social cognition and fear in humans. J Neurosci. 2005;25:11489-93.

14. Labuschagne I, Phan KL, Wood A, Angstadt M, Chua P, Heinrichs M, et al. Oxytocin attenuates amygdala reactivity to fear in generalized social anxiety disorder. Neuropsychopharmacology. 2010;35:2403-13.

15. Petrovic P, Kalisch R, Singer T, Dolan RJ. Oxytocin attenuates affective evaluations of conditioned faces and amygdala activity. J Neurosci. 2008;28:6607-15.

16. Riem MM, Bakermans-Kranenburg MJ, Pieper S, Tops M, Boksem MA, Vermeiren $\mathrm{RR}$, et al. Oxytocin modulates amygdala, insula, and inferior frontal gyrus responses to infant crying: a randomized controlled trial. Biol Psychiatry. 2011; 70:291-7.

17. Riem MM, van IJzendoorn MH, Tops M, Boksem MA, Rombouts SA, BakermansKranenburg MJ. No laughing matter: intranasal oxytocin administration changes functional brain connectivity during exposure to infant laughter. Neuropsychopharmacology. 2012;37:1257-66.

18. Singer T, Snozzi R, Bird G, Petrovic P, Silani G, Heinrichs M, et al. Effects of oxytocin and prosocial behavior on brain responses to direct and vicariously experienced pain. Emotion. 2008;8:781-91.

19. Baumgartner $T$, Heinrichs $M$, Vonlanthen $A$, Fischbacher $U$, Fehr E. Oxytocin shapes the neural circuitry of trust and trust adaptation in humans. Neuron. 2008:58:639-50.

20. Zunhammer M, Geis S, Busch V, Greenlee MW, Eichhammer P. Effects of intranasal oxytocin on thermal pain in healthy men: a randomized functional magnetic resonance imaging study. Psychosom Med. 2015;77:156-66.

21. Andari E, Richard N, Leboyer M, Sirigu A. Adaptive coding of the value of social cues with oxytocin, an fMRI study in autism spectrum disorder. Cortex. 2016; 76:79-88.

22. Watanabe $\mathrm{T}$, Abe $\mathrm{O}$, Kuwabara $\mathrm{H}$, Yahata N, Takano Y, Iwashiro N, et al. Mitigation of sociocommunicational deficits of autism through oxytocin-induced recovery of medial prefrontal activity: a randomized trial. JAMA Psychiatry. 2014;71:166-75.

23. Domes G, Lischke A, Berger C, Grossmann A, Hauenstein K, Heinrichs M, et al. Effects of intranasal oxytocin on emotional face processing in women. Psychoneuroendocrinology. 2010;35:83-93.

24. Pincus D, Kose S, Arana A, Johnson K, Morgan PS, Borckardt J, et al. Inverse effects of oxytocin on attributing mental activity to others in depressed and healthy subjects: a double-blind placebo controlled FMRI study. Front Psychiatry. 2010;1:134.

25. Rilling JK, DeMarco AC, Hackett PD, Thompson R, Ditzen B, Patel R, et al. Effects of intranasal oxytocin and vasopressin on cooperative behavior and associated brain activity in men. Psychoneuroendocrinology. 2012;37:447-61.

26. Wittfoth-Schardt D, Grunding J, Wittfoth M, Lanfermann $H$, Heinrichs $M$, Domes $G$, et al. Oxytocin modulates neural reactivity to children's faces as a function of social salience. Neuropsychopharmacology. 2012;37:1799-807.

27. Domes G, Heinrichs M, Kumbier E, Grossmann A, Hauenstein K, Herpertz SC. Effects of intranasal oxytocin on the neural basis of face processing in autism spectrum disorder. Biol Psychiatry. 2013;74:164-71.

28. Domes G, Kumbier E, Heinrichs M, Herpertz SC. Oxytocin promotes facial emotion recognition and amygdala reactivity in adults with asperger syndrome. Neuropsychopharmacology. 2014;39:698-706.

29. Seeley SH, Chou YH, O'Connor MF. Intranasal oxytocin and OXTR genotype effects on resting state functional connectivity: A systematic review. Neurosci Biobehav Rev. 2018;95:17-32.
30. Dodhia S, Hosanagar A, Fitzgerald DA, Labuschagne I, Wood AG, Nathan PJ, et al. Modulation of resting-state amygdala-frontal functional connectivity by oxytocin in generalized social anxiety disorder. Neuropsychopharmacology. 2014;39: 2061-9.

31. Ebner NC, Chen $H$, Porges $E$, Lin T, Fischer H, Feifel D, et al. Oxytocin's effect on resting-state functional connectivity varies by age and sex. Psychoneuroendocrinology. 2016;69:50-9.

32. Eckstein M, Markett S, Kendrick KM, Ditzen B, Liu F, Hurlemann R, et al. Oxytocin differentially alters resting state functional connectivity between amygdala subregions and emotional control networks: Inverse correlation with depressive traits. Neuroimage. 2017;149:458-67.

33. Koch SB, van ZM, Nawijn L, Frijling JL, Veltman DJ, Olff M. Intranasal oxytocin normalizes amygdala functional connectivity in posttraumatic stress disorder. Neuropsychopharmacology. 2016;41:2041-51.

34. Sripada CS, Phan KL, Labuschagne I, Welsh R, Nathan PJ, Wood AG. Oxytocin enhances resting-state connectivity between amygdala and medial frontal cortex. Int J Neuropsychopharmacol. 2013;16:255-60.

35. Kumar J, Vollm B, Palaniyappan L. Oxytocin affects the connectivity of the precuneus and the amygdala: a randomized, double-blinded, placebo-controlled neuroimaging trial. Int J Neuropsychopharmacol. 2014;18: pii: pyu051.

36. Fan Y, Herrera-Melendez AL, Pestke K, Feeser M, Aust S, Otte C, et al. Early life stress modulates amygdala-prefrontal functional connectivity: implications for oxytocin effects. Hum Brain Mapp. 2014;35:5328-39.

37. Riem MM, van IJzendoorn MH, Tops M, Boksem MA, Rombouts SA, BakermansKranenburg MJ. Oxytocin effects on complex brain networks are moderated by experiences of maternal love withdrawal. Eur Neuropsychopharmacol. 2013;23:1288-95.

38. Wigton $R$, Radua J, Allen $P$, Averbeck $B$, Meyer-Lindenberg A, McGuire $P$, et al. Neurophysiological effects of acute oxytocin administration: systematic review and meta-analysis of placebo-controlled imaging studies. J Psychiatry Neurosci. 2015;40:E1-22.

39. Dadds MR, MacDonald E, Cauchi A, Williams K, Levy F, Brennan J. Nasal oxytocin for social deficits in childhood autism: a randomized controlled trial. J Autism Dev Disord. 2014;44:521-31.

40. Guastella AJ, Gray KM, Rinehart NJ, Alvares GA, Tonge BJ, Hickie IB, et al. The effects of a course of intranasal oxytocin on social behaviors in youth diagnosed with autism spectrum disorders: a randomized controlled trial. J Child Psychol Psychiatry. 2015;56:444-52.

41. Anagnostou E, Soorya L, Chaplin W, Bartz J, Halpern D, Wasserman S, et al. Intranasal oxytocin versus placebo in the treatment of adults with autism spectrum disorders: a randomized controlled trial. Mol Autism. 2012;3:16.

42. Parker KJ, Oztan O, Libove RA, Sumiyoshi RD, Jackson LP, Karhson DS, et al. Intranasal oxytocin treatment for social deficits and biomarkers of response in children with autism. Proc Natl Acad Sci USA. 2017;114:8119-24.

43. Watanabe T, Kuroda M, Kuwabara H, Aoki Y, Iwashiro N, Tatsunobu N, et al. Clinical and neural effects of six-week administration of oxytocin on core symptoms of autism. Brain. 2015;138:3400-12.

44. Yatawara CJ, Einfeld SL, Hickie IB, Davenport TA, Guastella AJ. The effect of oxytocin nasal spray on social interaction deficits observed in young children with autism: a randomized clinical crossover trial. Mol Psychiatry. 2015;21: 1225-31.

45. Alaerts K, Bernaerts S, Vanaudenaerde B, Daniels N, Wenderoth N. Amygdalahippocampal connectivity is associated with endogenous levels of oxytocin and can be altered by exogenously administered oxytocin in adults with autism. Biol Psychiatry Cogn Neurosci Neuroimaging. 2019;4:655-63.

46. Bernaerts S, Boets B, Bosmans G, Steyaert J, Alaerts K. Behavioral effects of multiple-dose oxytocin treatment in autism: a randomized, placebo-controlled trial with long-term follow-up. Mol Autism. 2020;11:6.

47. Lord C, Rutter M, DiLavore PC, Risi S. Autism Diagnostic Observation Schedule. Los Angeles: Western Psychological Service; 1999.

48. Bos PA, Panksepp J, Bluthe RM, van HJ. Acute effects of steroid hormones and neuropeptides on human social-emotional behavior: a review of single administration studies. Front Neuroendocrinol. 2012;33:17-35.

49. Paloyelis Y, Doyle OM, Zelaya FO, Maltezos S, Williams SC, Fotopoulou A, et al. A spatiotemporal profile of in vivo cerebral blood flow changes following intranasal oxytocin in humans. Biol Psychiatry. 2016;79:693-705.

50. Constantino JN, Davis SA, Todd RD, Schindler MK, Gross MM, Brophy SL, et al. Validation of a brief quantitative measure of autistic traits: comparison of the social responsiveness scale with the autism diagnostic interview-revised. J Autism Developmental Disord. 2003;33:427-33.

51. Lam KS, Aman MG. The repetitive behavior scale-revised: independent validation in individuals with autism spectrum disorders. J Autism Dev Disord. 2007;37:855-66.

52. Gillath O, Hart J, Noftle EE, Stockdale GD. Development and validation of a state adult attachment measure (SAAM). J Res Personal. 2009;43:362-73. 
Oxytocin induces long-lasting adaptations within amygdala circuitry in... $\mathrm{K}$ Alaerts et al.

53. Lahnakoski JM, Glerean E, Salmi J, Jaaskelainen I, Sams M, Hari R, et al. Naturalistic fMRI mapping reveals superior temporal sulcus as the hub for the distributed brain network for social perception. Front Hum Neurosci. 2012;6:233.

54. LeDoux JE, Iwata J, Cicchetti P, Reis DJ. Different projections of the central amygdaloid nucleus mediate autonomic and behavioral correlates of conditioned fear. J Neurosci. 1988;8:2517-29.

55. Grace SA, Rossell SL, Heinrichs M, Kordsachia C, Labuschagne I. Oxytocin and brain activity in humans: a systematic review and coordinate-based meta-analysis of functional MRI studies. Psychoneuroendocrinology. 2018;96:6-24.

56. Wang D, Yan X, Li M, Ma Y. Neural substrates underlying the effects of oxytocin: a quantitative meta-analysis of pharmaco-imaging studies. Soc Cogn Affect Neurosci. 2017;12:1565-73.

57. Alaerts K, Woolley DG, Steyaert J, Di MA, Swinnen SP, Wenderoth N. Underconnectivity of the superior temporal sulcus predicts emotion recognition deficits in autism. Soc Cogn Affect Neurosci. 2014;9:1589-600.
58. Pelphrey KA, Shultz S, Hudac CM, Wyk BCV. Research review: constraining heterogeneity: the social brain and its development in autism spectrum disorder. $J$ Child Psychol Psychiatry. 2011;52:631-44.

59. Ma Y, Shamay-Tsoory S, Han S, Zink CF. Oxytocin and social adaptation: insights from neuroimaging studies of healthy and clinical populations. Trends Cogn Sci. 2016;20:133-45.

60. Fatemi SH, Aldinger KA, Ashwood P, Bauman ML, Blaha CD, Blatt GJ, et al. Consensus paper: pathological role of the cerebellum in autism. Cerebellum. 2012;11:777-807.

61. Kovacs B, Keri S. Off-label intranasal oxytocin use in adults is associated with increased amygdala-cingulate resting-state connectivity. Eur Psychiatry. 2015;30:542-7.

62. van IJzendoorn MH, Bhandari R, van d V, Grewen KM, Bakermans-Kranenburg MJ. Elevated salivary levels of oxytocin persist more than $7 \mathrm{~h}$ after intranasal administration. Front Neurosci. 2012;6:174. 